\title{
On the ferromagnetic ground state of $\mathrm{SmN}$
}

\author{
J. F. McNulty, B. J. Ruck, and H. J. Trodahl \\ The MacDiarmid Institute for Advanced Materials and Nanotechnology, School of Chemical and Physical Sciences, \\ Victoria University of Wellington, P.O. Box 600, Wellington, New Zealand
}

(Dated: March 6, 2018)

\begin{abstract}
$\mathrm{SmN}$ is a ferromagnetic semiconductor with the unusual property of an orbital-dominant magnetic moment that is largely cancelled by an antiparallel spin contribution, resulting in a near-zero net moment. However, there is a basic gap in the understanding of the ferromagnetic ground state, with existing density functional theory calculations providing values of the $4 f$ magnetic moments at odds with experimental data. To clarify the situation, we employ an effective $4 f$ Hamiltonian incorporating spin-orbit coupling, exchange, the crystal field, and $J$-mixing to calculate the ground state $4 f$ moments. Our results are in excellent agreement with experimental data, revealing moderate quenching of both spin and orbital moments to magnitudes of $\sim 2 \mu_{B}$ in bulk $\mathrm{SmN}$, enhanced to an average of $\sim 3 \mu_{B}$ in $\mathrm{SmN}$ layers within a $\mathrm{SmN} / \mathrm{GdN}$ superlattice. These calculations provide insight into recent studies of $\mathrm{SmN}$ showing that it is an unconventional superconductor at low temperatures and displays twisted magnetization phases in magnetic heterostructures.
\end{abstract}

\section{INTRODUCTION}

In recent years, research into $\mathrm{SmN}$ has been driven by two main considerations; its potential for spintronic applications and its vanishingly small ferromagnetic moment. It is a member of the rare-earth nitride series, a group of materials owing their promise for spintronic applications to their status as intrinsic ferromagnetic semiconductors 1010. SmN is especially unique among the series, with a vanishingly small ordered moment arising from the near-cancellation of spin and orbital moments [4, 5] fixed antiparallel by strong spin-orbit coupling. So far the spintronic potential of the RENs remains largely untapped, though GdN has been integrated within spinfilters [11, 12] and field effect transistors [13] while DyN has been incorporated into a magnetic tunnel junction [14. However, SmN has quite recently displayed remarkable phenomena in magnetization and transport studies. There is a report of an unconventional twisted magnetization phase occurring in SmN films exchange coupled to $\mathrm{GdN}$, observed via x-ray magnetic circular dichroism (XMCD) 15, and the discovery of superconductivity coexisting with ferromagnetic order at temperatures of 3$5 \mathrm{~K}$ in $\mathrm{SmN}$ films and $\mathrm{SmN} / \mathrm{GdN}$ superlattices, thought to be due to a spin-triplet pairing mechanism [16]. These phenomena are intimately related to the ferromagnetic state of $\mathrm{SmN}$, which displays ferromagnetic order below $T_{C} \approx 27 \mathrm{~K}$ with a near-zero moment of $0.035 \mu_{B}$ per $\mathrm{Sm}^{3+}$, with the unusual situation that the orbital moment is marginally dominant, and defines the net magnetization direction [4, 5, 17].

The magnetic state of $\mathrm{SmN}$ originates from the partially filled $4 f$ shell of the trivalent Sm ion, the usual valence among the RENs except $\mathrm{CeN}$ and $\mathrm{EuN}$ [1, 7, 8, 18, with Hund's rules providing the ground state configuration. In $\mathrm{Sm}^{3+}\left(4 f^{5}\right)$, Hund's rules yield a $4 f$-shell spin alignment of $S=5 / 2$, with the spin-orbit coupling enforcing an opposed orbital alignment of $L=5$, re- sulting in a total angular momentum quantum number $J=|L-S|=5 / 2$ and Landé factor of $g_{J}=2 / 7$. The $J=5 / 2$ ground state-multiplet of $\mathrm{Sm}^{3+}$ is well-known to be influenced by the first excited $J=7 / 2$ multiplet which is separated by $\Delta E \approx 1500 \mathrm{~K}$ from the ground state [5, 19, 20]. However, the effect of this excited state is not large enough to explain the discrepancy between the experimentally measured $\mathrm{SmN}$ moment of $0.035 \mu_{B}$ and the theoretical free-ion saturation moment of the groundstate multiplet $\mu_{B}\left\langle L_{z}+2 S_{z}\right\rangle_{G S}=\mu_{B} g_{J} J=0.74 \mu_{B}$ 5]. The experimental $0.45 \mu_{B}$ paramagnetic moment is almost half the free-ion value of $\mu_{B} g_{J} \sqrt{J(J+1)}=$ $0.85 \mu_{B}$, but is close to the $0.41 \mu_{B}$ moment expected for a $\Gamma_{7}$ crystal-field ground state doublet $[5$, signalling that the crystal field may also play an important role in the small ferromagnetic moment. The low Curie temperature of $\approx 27 \mathrm{~K}$ in $\mathrm{SmN}$ fits in with the trend of low ordering temperatures in the REN series [1] which is due to weak indirect exchange. The highly localized $4 f$ states ensure that the inter-ionic exchange mechanism precipitating ferromagnetism proceeds via $\mathrm{Sm} 5 d$ and N $2 p$ states in indirect exchange processes [1, 21, 22. Because of the semiconducting nature of the RENs, the carrier-mediated RKKY mechanism does not appear to be the dominant exchange channel as it is in metallic rare-earth systems 23. 24.

To date there has been no convincing description of $\mathrm{SmN}$ which accounts for both the magnitude and orbitaldominant sign of the ordered moment. Furthermore, XMCD experiments on bulk $\mathrm{SmN}$ and a $\mathrm{SmN} / \mathrm{GdN}$ superlattice show a $60 \%$ enhancement in the magnitude of the Sm $4 f$ polarization in the superlattice compared to bulk SmN, caused by interface exchange with highly spinpolarized GdN [15, 17]. This implies that the bulk Sm $4 f$ spin $\left(m_{S}^{4 f}\right)$ and orbital $\left(m_{L}^{4 f}\right)$ moments are not fully polarized $\left(=5 \mu_{B}\right)$, but are significantly reduced from full alignment, consistent with the $4 f$ electrons being dominated by spin-orbit coupling and crystal field energies. 
Only two calculations providing values of the $\mathrm{SmN}$ magnetic moment are available, both of which use density functional theory in the LSDA $+U$ approximation. A report by Larson et al. 25] calculated a ferromagnetic ground state moment nearly equal to the experimental value, with the opposing $4 f$ spin and orbital moments of magnitude $\approx 4.9 \mu_{B}$, while the $5 d$ and $2 p$ states provided moments of order $0.1 \mu_{B}$ but with opposite sign. However, the net moment was found to be parallel to the spin moment (i.e., spin-dominant). Another study by Morari et al. 26] used a variety of LSDA $+U$ implementations and parameters to find even larger values for the net moment, though these were also spin-dominant. The ground state was furthermore antiferromagnetic, contradicting recent experiments unambiguously displaying ferromagnetism [5, 17. Other band structure calculations on EuN [7] and TmN 27] suggest that dynamical meanfield theory in the Hubbard- $I$ approximation provided better agreement with experiment than $\mathrm{LSDA}+U$, suggesting alternative techniques should be explored.

Here we pursue an alternative to band structure calculations to explain experimental situation in $\mathrm{SmN}$ by directly diagonalizing an effective $4 f$ Hamiltonian to yield spin and orbital moments of the ground state. We neglect conduction electron contributions which are small for semiconducting $\mathrm{SmN}$, serving only as a weak correction to our results. We employ a model of the $\mathrm{Sm}^{3+}$ ion in a cubic crystal field, incorporating excited multiplets following DeWijn et al. 19, exchange in a self-consistent mean-field approach [20], and spin-orbit coupling. The results demonstrate that the small orbital-dominant ferromagnetic moment of $\mathrm{SmN}$ naturally emerges from partial-quenching of both orbital and spin $4 f$ moments, linked together by the spin-orbit coupling. The moments were calculated to be less than half of the maximum $5 \mu_{B}$ polarization of the $L S$-coupled state. We also demonstrate a correspondence between the $5 d$ polarization measured by XMCD and the mean-field exchange constant, and demonstrate that the enhanced $4 f$ and $5 d$ XMCD polarization observed in a $\mathrm{SmN} / \mathrm{GdN}$ superlattice compared to bulk SmN can be understood within our model. These results provide the first description of the $4 f$ magnetic state of $\mathrm{SmN}$ in quantitative agreement with the various experimental results and provide crucial insight into recent experiments showing unconventional twisted magnetization phases and triplet superconductivity in $\mathrm{SmN}$.

\section{CALCULATION PROCEDURE}

The magnetic properties of $\mathrm{SmN}$ are primarily dictated by the $\mathrm{Sm}^{3+}$ ion, with its atomic-like $4 f$ orbitals. Examples of of $\mathrm{Sm}^{3+}$ compounds studied in the past include metallic Laves-phase compounds such as $\mathrm{SmAl}_{2}$, $\mathrm{Sm}_{1-x} \mathrm{Gd}_{x} \mathrm{Al}_{2}$, and $\mathrm{Sm}_{1-x} \mathrm{Nd}_{x} \mathrm{Al}_{2}$ [19, 28, 33, CsCl-type structure SmZn and SmCd [34, and Sm metal [20]. These studies all demonstrate that the magnetic properties of trivalent Sm compounds are strongly influenced by the first excited $J=7 / 2$ multiplet in addition to the ground $J=5 / 2$ multiplet. There are two reasons for this: 1) the exchange and Zeeman terms along with the crystal field of the surrounding anions cause admixtures of the ground and excited states, and 2) the small ground state Landé $g_{J}$-factor of $\frac{2}{7}$ for $\mathrm{Sm}^{3+}$ means the matrix elements within the $J=5 / 2$ multiplet are small compared to $\Delta J=1$ matrix elements. An important consequence is the zero-moment ferromagnetism observed in Gd doped $\mathrm{SmAl}_{2}$ [30, 33. In that compound, the $J$-mixing of the ground and first excited multiplets causes different temperature dependencies of $m_{S}^{4 f}$ and $m_{L}^{4 f}$, resulting in a transition from orbital- to spin-dominant magnetism at a compensation temperature where the net moment is zero, while long-range ferromagnetic order is still present.

Crystal field calculations of magnetic properties in rare-earth systems are usually described within the Steven's formalism of operator equivalents [35, 36], however these techniques are insufficient for $\mathrm{Sm}^{3+}$ as they only consider the ground state $J$-multiplet. Here we use an extension of the Steven's formalism which allows for the inclusion of higher multiplets [19], derived with the algebraic methods of tensor operators.

The effective $4 f$ Hamiltonian from which we can calculate the ground-state expectation values includes the spin-orbit coupling $\left(\mathcal{H}_{S O}\right)$, exchange interaction $\left(\mathcal{H}_{e x}\right)$, and crystal field $\left(\mathcal{H}_{C F}\right)$. We ignore the Zeeman coupling for calculation of the spontaneous moment. Including the $4 f$ inter-ion exchange in a self-consistent mean-field approach [20, 34], the Hamiltonian is

$$
\mathcal{H}=\Lambda \mathbf{L} \cdot \mathbf{S}-\mathcal{J}\left\langle S_{z}\right\rangle S_{z}+\mathcal{H}_{C F},
$$

where the spin-orbit coupling constant $\Lambda$ is approximately $430 \mathrm{~K}$, calculated from a knowledge of the energy splittings between $J$-multiplets, expressed as $\Delta E=$ $E_{J+1}-E_{J}=\Lambda(J+1) \approx 1500 \mathrm{~K}$ [5, 34]. The second term in Eq. 1 accounts for exchange, where $\mathcal{J}$ is the effective exchange constant and $\left\langle S_{z}\right\rangle$ is the expectation value of the spin operator. The exchange constant can be estimated from the paramagnetic Curie temperature $\Theta$, which in the mean-field approximation is given by

$$
\Theta=2 \mathcal{J}\left(g_{J}-1\right)^{2} J(J+1) / 3 k_{B} .
$$

The de Gennes factor $G=\left(g_{J}-1\right)^{2} J(J+1)$ of the ground state multiplet linking the exchange constant and $\Theta$ is usually found to describe the trend in $\Theta$ versus $G$ in iso-structural rare-earth compounds. However, this relation ignores the influence of $J$-mixing by the exchange field, and thus slightly overestimates $\mathcal{J}$. By considering the van Vleck temperature-independent contribution to the susceptibility, one can derive [see Appendix] the first- 
order correction to the calculation of $\mathcal{J}$ as

$$
\mathcal{J}=\frac{3}{2} \frac{k_{B} \Theta}{\left(g_{J}-1\right)^{2} J(J+1)}\left[\frac{1}{1+12 k_{B} \Theta / \Delta E}\right] .
$$

Among the rare-earths this correction factor is only significant for $\mathrm{Sm}$ and $\mathrm{Eu}$ ions, and in the case of $\mathrm{Sm}^{3+}$ it gives a correction factor of $\left[1+12 k_{B} \Theta / \Delta E\right]^{-1}=0.83$, yielding $\mathcal{J}=7.9 \mathrm{~K}$. While the correction here does not qualitatively alter the results of the following calculations, we include it to demonstrate the influence of $J$ mixing on the mean-field exchange constant.

The rock-salt structure of $\mathrm{SmN}$ results in cubic octahedral coordination of the $\mathrm{Sm}^{3+}$ ion, and we choose the $z$ axis to be along the [111] direction [37. The crystal field Hamiltonian is then given by [19, 36.

$$
\begin{aligned}
\mathcal{H}_{C F}= & -\frac{2}{3} A_{4} \sum_{i}\left(f_{40}\left(\mathbf{r}_{i}\right)-20 \sqrt{2} f_{43}\left(\mathbf{r}_{i}\right)\right) \\
& +\frac{16}{9} A_{6} \sum_{i}\left(f_{60}\left(\mathbf{r}_{i}\right)+\frac{35 \sqrt{2}}{4} f_{63}\left(\mathbf{r}_{i}\right)+\frac{77}{8} f_{66}\left(\mathbf{r}_{i}\right)\right),
\end{aligned}
$$

where the tesseral harmonics $f_{k q}\left(\mathbf{r}_{i}\right)$ are renormalized and purely real combinations of spherical harmonics 36 . The parameters $A_{4}$ and $A_{6}$ are the fourth- and sixthorder crystal field parameters discussed below, while the sum is over the five $4 f$ electrons. An arbitrary matrix element of $\mathcal{H}_{C F}$ has the form

$$
\left\langle J^{\prime} M_{J^{\prime}}\left|\mathcal{H}_{C F}\right| J M_{J}\right\rangle=\chi_{4} A_{4}\left\langle r^{4}\right\rangle+\chi_{6} A_{6}\left\langle r^{6}\right\rangle,
$$

where $\chi_{k}$ contain the angular part of the matrix elements, described in Ref. [19] for all the trivalent rare-earth ions. We are then left to determine $A_{4}\left\langle r^{4}\right\rangle$ and $A_{6}\left\langle r^{6}\right\rangle$, which can be either treated as adjustable parameters or calculated within, e.g., the point-charge model.

After diagonalization of equation (1), the magnetic moment in the ground state $(T=0)$ is defined by the expectation value

$$
m=\mu_{B}\left\langle L_{z}+2 S_{z}\right\rangle,
$$

where $\langle\cdots\rangle$ is taken within the ground-state, $|\psi\rangle_{G S}=$ $\sum_{J} \sum_{M_{J}} c_{J, M_{J}}\left|J M_{J}\right\rangle$. Similarly we have $m_{L}^{4 f}=\mu_{B}\left\langle L_{z}\right\rangle$ and $m_{S}^{4 f}=2 \mu_{B}\left\langle S_{z}\right\rangle$. We note that in Eq. (6) and for the individual moments we use the opposite sign convention to define the magnetic moment compared with the standard definition. If we restrict to the $J=5 / 2$ multiplet, the matrix elements are given by $\left\langle J M_{J}\left|L_{z}+2 S_{z}\right| J M_{J}\right\rangle=g_{J} J$, with $\left\langle L_{z}\right\rangle=\left(2-g_{J}\right) J$ and $\left\langle S_{z}\right\rangle=\left(g_{J}-1\right) J$. If we consider instead the lowest three $J=5 / 2,7 / 2,9 / 2$ multiplets, the Hamiltonian has dimension $24 \times 24$, and the matrix elements of $L_{z}, S_{z}$ and $L_{z}+2 S_{z}$ can be calculated in terms of Wigner $3 j$ and $6 j$ symbols, as given in Ref. [19. When the exchange term

\begin{tabular}{lcccc}
\hline$J$-multiplets & $\mathcal{J}$ & $\left\langle S_{z}\right\rangle$ & $\left\langle L_{z}\right\rangle$ & $\left\langle L_{z}+2 S_{z}\right\rangle$ \\
\hline \hline$\frac{5}{2}$ & 0 & -1.786 & 4.286 & 0.714 \\
$\frac{5}{2}, \frac{7}{2}$ & 0 & -1.786 & 4.286 & 0.714 \\
$\frac{5}{2}, \frac{7}{2}$ & $7.9 \mathrm{~K}$ & -1.852 & 4.352 & 0.648 \\
\hline
\end{tabular}

TABLE I. Expectation values of the angular momentum operators for the $\mathrm{Sm}^{3+}$ ion based on number of $J$-multiplets included, only considering the spin-orbit coupling and exchange (i.e. omitting the crystal field).

is included, an initial guess of $\left\langle S_{z}\right\rangle$ is made, $\mathcal{H}$ is diagonalized, and the resulting ground-state expectation value $\left\langle S_{z}\right\rangle$ is then reinserted directly into Eq. (1) repeatedly until convergence is reached, typically requiring $N \sim 10$ or less iterations.

The effect of including higher multiplets can be seen in Table 1, where only spin-orbit coupling and exchange are considered. The free-ion moments $(\mathcal{J}=0)$ yield orbital to spin ratio of $m_{L}^{4 f} / m_{S}^{4 f}=-1.2$, while the inclusion of the ferromagnetic exchange term with $\mathcal{J}=7.9 \mathrm{~K}$ reduces the ratio to $m_{L}^{4 f} / m_{S}^{4 f}=-1.175$, due to the off-diagonal terms in $\mathcal{H}_{e x}$ mixing in the excited multiplet (inclusion of the $J=9 / 2$ multiplet returns the same expectation values to the given precision). The small reduction in the net moment due to the weak exchange interaction in $\mathrm{SmN}$ is clearly unable to account for the experimental moment of $0.035 \mu_{B}$.

We note that if $\mathcal{H}_{S O}$ is treated as a small perturbation to $\mathcal{H}_{e x}$, then the net moment is reduced to zero when higher multiplets are included because $L_{z}$ and $S_{z}$ take on fixed values of $M_{L}$ and $M_{S}$ (e.g., $L_{z}|\psi\rangle=M_{L}|\psi\rangle$ ) for which the ground state has $M_{L}=5$ and $M_{S}=-5 / 2$, yielding zero net moment. These values of $m_{S}^{4 f}$ and $m_{L}^{4 f}$ are close to what is found in the LSDA $+U$ calculations of Ref. [25], with $m_{L}^{4 f} / m_{S}^{4 f}=-4.85 / 4.91=-0.98$. In addition, Ref. 26, using various implementations of $\mathrm{LSDA}+U$ and a range of parameters, calculated $\mathrm{SmN}$ values in the range of $m_{L}^{4 f} / m_{S}^{4 f}=-4.57 / 5.00=-0.85$ to $m_{L}^{4 f} / m_{S}^{4 f}=-3.46 / 4.09=-0.91$. All of these LSDA $+U$ calculations show a spin-dominant $\mathrm{SmN}$ moment, in contrast to the orbital dominant moment of both the free ion and $\mathrm{SmN}$.

\section{RESULTS}

Figure 1 shows the resulting ground-state expectation value $\mu_{B}\left\langle L_{z}+2 S_{z}\right\rangle$, plotted for crystal field parameters $A_{4}\left\langle r^{4}\right\rangle, A_{6}\left\langle r^{6}\right\rangle \in[-350 \mathrm{~K},+350 \mathrm{~K}]$ with contours of constant $\mu_{B}\left\langle L_{z}+2 S_{z}\right\rangle$ plotted, including a dashed contour corresponding to a calculated net moment equal to the experimental moment of $0.035 \mu_{B}$. The ground state moment was found by diagonalizing the Hamiltonian in Eq. (1) within the three lowest multiplets $(J=5 / 2,7 / 2,9 / 2)$, with $\mathcal{J}=7.9 \mathrm{~K}$. To limit the range 


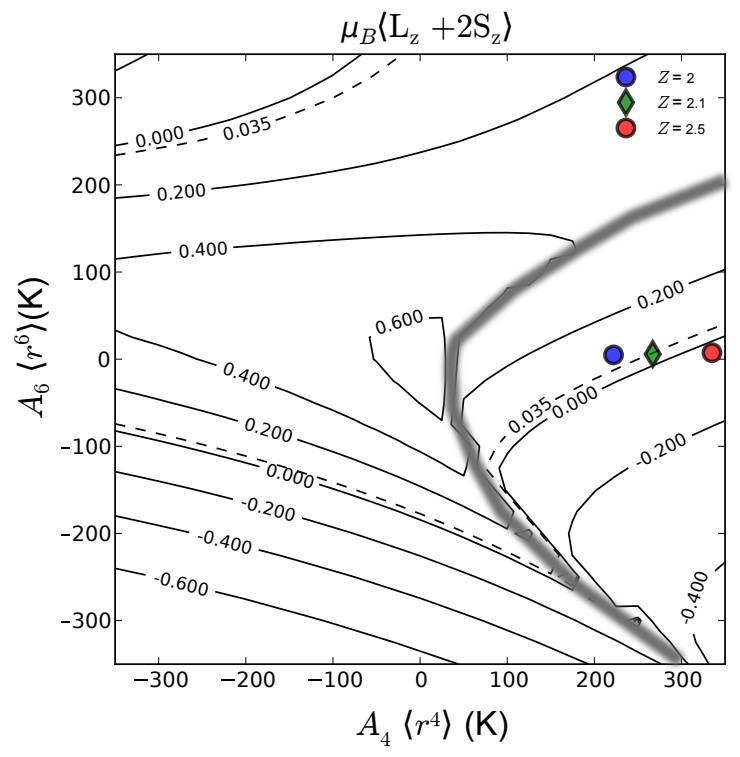

FIG. 1. Contours of $\mu_{B}\left\langle L_{z}+2 S_{z}\right\rangle$ for the fourth and sixth order crystal field parameters $\left(\left\langle L_{z}\right\rangle\right.$ is taken as positive). The dashed contour corresponds to $\mu_{B}\left\langle L_{z}+2 S_{z}\right\rangle=0.035 \mu_{B}$, equal to the experimental net moment. The heavy grey line demarcates the the boundary of the $\Gamma_{7}$ doublet and $\Gamma_{8}$ quartet, the area to the right of this line has a $\Gamma_{7}$ ground state. The symbols represent the net $4 f$ moment returned using crystal field parameters calculated within the point-charge model for three values of $Z$.

of crystal field parameters producing agreement with the experimental data in Fig. 11 we must impose some restrictions. One restriction is provided by experimental results revealing an effective paramagnetic moment in $\mathrm{SmN}$ close to that calculated from a $\Gamma_{7}$ ground-state doublet [5]. The $\Gamma_{7}$ ground state corresponds to the region to the right of the heavy line demarcating where the $\Gamma_{8}$ quartet and $\Gamma_{7}$ doublet are degenerate, restricting the possible crystal field parameters to within this area.

A further refinement of the crystal field parameters can be made using the the electric-point charge approximation, which allows us to parametrize the crystal field strength in terms of a single effective charge $Z$. While this is a phenomenological approach, it has been shown to provide reasonable agreement with the variation in crystal field strength across the light rare-earth pnictides $(\mathrm{P}, \mathrm{As}, \mathrm{Sb}, \mathrm{Bi})[38$ as well as some of the other rare-earth nitrides [39], and can thus provide valuable insight where more sophisticated techniques have failed. In the point charge model, the coefficients $A_{4}$ and $A_{6}$ are given by the following expressions in the case of an octahedrally coordinated rare-earth ion [36, 40,

$$
A_{4}=\frac{7}{16} \frac{Z e^{2}}{R^{5}}, \quad A_{6}=\frac{3}{64} \frac{Z e^{2}}{R^{7}},
$$

where $-Z|e|$ is the effective charge of each $\mathrm{N}$ ligand, and $R$ is the separation between the $\mathrm{Sm}$ ion and $\mathrm{N}$, given by half of the experimental $\mathrm{SmN}$ lattice-constant $R=$

\begin{tabular}{|c|c|c|c|c|c|}
\hline \multicolumn{6}{|c|}{$J=5 / 2,7 / 2,9 / 2$ included } \\
\hline $\mathcal{J}(\mathrm{K})$ & $Z \quad A_{4}(\mathrm{~K})$ & $A_{6}(\mathrm{~K})$ & $\left\langle S_{z}\right\rangle$ & $\left\langle L_{z}\right\rangle$ & $\left\langle L_{z}+2 S_{z}\right.$ \\
\hline 7.9 & 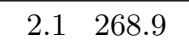 & 5.9 & -0.976 & 1.988 & 0.036 \\
\hline $\mathcal{J}_{S L}=24.5$ & $5 \quad 2.1 \quad 268.9$ & 5.9 & -1.48 & 3.11 & 0.16 \\
\hline \multicolumn{6}{|c|}{$J=5 / 2$ only } \\
\hline $\mathcal{J}(\mathrm{K}) 2$ & 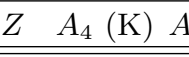 & $A_{6}(\mathrm{~K})$ & $\left\langle S_{z}\right\rangle \quad\langle L$ & $\left.L_{z}\right\rangle\left\langle L_{z}\right.$ & $\left.z+2 S_{z}\right\rangle$ \\
\hline 7.9 & $2.1 \quad 268.9$ & $5.9-$ & -0.701 .6 & 69 & 0.28 \\
\hline
\end{tabular}

TABLE II. (Top) Expectation values of magnetic moments in $\mathrm{SmN}$ with crystal field parameters calculated in the point charge model. (Bottom) Expectation values only considering the ground $J=5 / 2$ multiplet.

$a / 2=2.52 \AA$ [1]. The radial integrals $\left\langle r^{k}\right\rangle$ have been calculated in various approaches, notably non-relativistic Hartree-Fock values [41] and relativistic Dirac-Fock calculations [42]. The latter of these yield for $\mathrm{Sm}^{3+}$ the values $\left\langle r^{4}\right\rangle=2.26 a_{0}^{6}$ and $\left\langle r^{6}\right\rangle=10.55 a_{0}^{6}$, where $a_{0}=0.529 \AA$ is the Bohr radius [43]. We are then left to determine the effective charge $Z$ of the $\mathrm{N}$ anions. For example, a value of $Z=1.6$ was found to explain the trend in the fourth order crystal field parameter in the light rare-earth pnictides (scaled to Dirac-Fock values) 38, while larger values of $Z$ are common in a few of the other rare-earth nitrides, ranging from 2.3 to 3.6 39. This difference between the rare-earth nitrides and the pnictides originates in the greater electronegativity of nitrogen (and hence more ionic behavior), resulting in the semiconducting nature of the nitrides, while the other pnictides are metallic.

In Figure 2 we present ground-state expectation values calculated using the point charge model with various values of $Z$ to parametrize the crystal field strength. The expectation values are plotted against $\mathcal{J}$, centered around the experimentally-derived value of $\mathcal{J}=7.9 \mathrm{~K}$, in order to show the influence of exchange. The first panel of Fig. 2 2 shows that the experimental moment of $0.035 \mu_{B}$ is reproduced by using parameters obtained from the pointcharge model with $Z=2.1$ and $\mathcal{J}=7.9 \mathrm{~K}$ (see also Table III. The value of $Z=2.1$ fits in with the trends observed in the rare-earth nitrides and pnictides, larger than the $Z=1.6$ value found for the light rare-earth pnictides and slightly less than the $Z=2.3-3.6$ range found in a number of rare-earth nitrides, as noted above. This result demonstrates that using reasonable parameters, the model reproduces the sign and magnitude of the experimental net moment of SmN. Furthermore, in Figure 2 (b) we see that the spin and orbital moments have magnitudes of $\approx 2 \mu_{B}$, strongly quenched compared to both the free-ion moments and the values given in Refs. 25, 26]

Figure2 (b) also shows that for $Z=2.1$ and $\mathcal{J}=7.9 \mathrm{~K}$ the ratio $m_{L}^{4 f} / m_{S}^{4 f}=-1.02$ is suppressed from the freeion value of -1.2 . This suppressed ratio only occurs when there is $J$-mixing. For example, if we are restricted to 

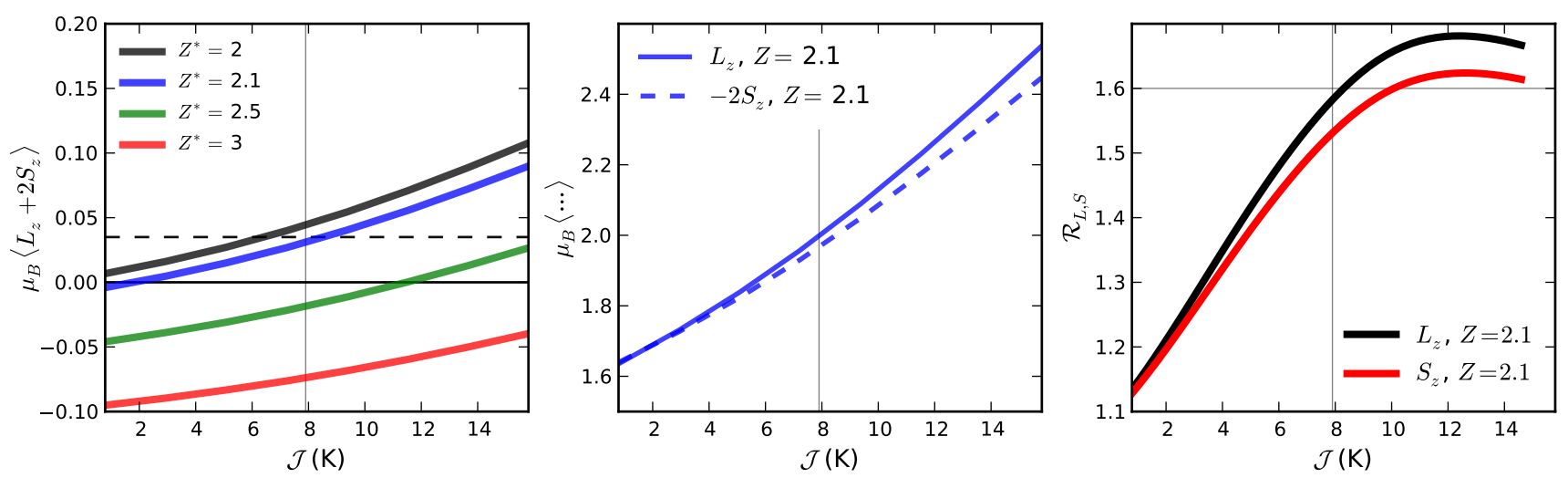

FIG. 2. (a) The ground state $4 f$ expectation value $\left\langle L_{z}+2 S_{z}\right\rangle$ calculated within the lowest three $J$-multiplets for various values of $Z$, with $\left\langle L_{z}\right\rangle$ taken as positive. (b) Expectation values $\left\langle L_{z}\right\rangle$ and $-2\left\langle S_{z}\right\rangle$ for $Z=2.1$. (c) The parameters $\mathcal{R}_{L}=$ $\left\langle L_{z}\right\rangle_{\mathcal{J}_{S L}} /\left\langle L_{z}\right\rangle_{\mathcal{J}}$ and $\mathcal{R}_{S}=\left\langle S_{z}\right\rangle_{\mathcal{J}_{S L}} /\left\langle S_{z}\right\rangle_{\mathcal{J}}$ representing the enhancement in the superlattice.

the ground-state $J$-multiplet, the Wigner-Eckart theorem assures that the ratio $\left\langle L_{z}\right\rangle /\left\langle 2 S_{z}\right\rangle=\left(2-g_{J}\right) /\left[2\left(g_{J}-1\right)\right]=$ -1.2 is fixed and independent of the parameters in the Hamiltonian. From this point of view we can see that the net moment is strongly influenced not only by the overall reduction of the spin and orbital moments, but also by their changing ratio due to $J$-mixing.

While the net $4 f$ moment calculated above can be compared to the experimental net moment (ignoring other contributions), it is more difficult to obtain experimental values of $m_{S}^{4 f}$ and $m_{L}^{4 f}$. The ideal method is through x-ray magnetic circular dichroism (XMCD) at the rareearth M-edge, which is in principle able to determine $m_{S}^{4 f}$ and $m_{L}^{4 f}$ experimentally via the XMCD sum rules for electric-dipole transitions [44, 45]. Experimental limitations have so far prevented a quantitative sum rule analysis in $\mathrm{SmN}$, but we may still extract meaningful conclusions by exploring the $\mathrm{Sm} \mathrm{L}_{3}$-edge XMCD measured in a $\mathrm{SmN}$ film and a $\mathrm{SmN} / \mathrm{GdN}$ superlattice, reported in Ref. [15].

Figure 3 shows the $\mathrm{Sm} \mathrm{L}_{3}$ edge XMCD data taken from Ref. [15], which showing the spectra from a bulk SmN sample and a $12 \times[1.5 \mathrm{~nm} \mathrm{SmN/9} \mathrm{nm} \mathrm{GdN]} \mathrm{super-}$ lattice. The $\mathrm{Sm} \mathrm{L}_{3}$ edge is dominated by electric-dipole (ED) $(2 p \rightarrow 5 d)$ transitions at high energy. These ED transitions provide information on the sign and magnitude of the polarization of the unoccupied Sm $5 d$ states which mediate exchange between the localized $4 f$ moments. At lower energy, the electric-quadrupole (EQ) transitions $(2 p \rightarrow 4 f)$ are visible, providing sign and magnitude information on the $4 f$ shell alignment. To a good approximation, the integral over the $\mathrm{Sm} \mathrm{L}_{3} \mathrm{EQ}$ spectrum scales linearly with $\left\langle S_{z}\right\rangle$ and $\left\langle L_{z}\right\rangle$, which can be seen from the XMCD sum rules derived for EQ transitions [46. As discussed in Refs [15, 17, the sign change between spectra indicates the magnetization of the thin SmN layers are forced into alignment with the GdN by strong interface-exchange in the superlattice. In addition to the sign change, the ED signal is enhanced by a factor of 3.1 in the superlattice, while the EQ feature is only enhanced by a factor of 1.6 .

First, we point out that an enhancement of the EQ signal in the superlattice $\mathrm{SmN}$ layers indicates that the $4 f$ spin and orbital moments cannot be maximal $\left(=5 \mu_{B}\right)$ in the bulk SmN, as this would preclude any enhancement. If we assume the superlattice $\mathrm{SmN}$ has spin/orbital moments maximized at $5 \mu_{B}$, an upper bound on the bulk SmN spin/orbital moments can be determined as $\left|m_{L, S}^{4 f, \max }\right| \approx 5 \mu_{B} / 1.6=3.1 \mu_{B}$. This upper bound is already well below the LSDA $+U$ results of Refs. [25, 26], but is still larger than the present calculations which show moments of magnitude $\approx 2 \mu_{B}$. Clearly, even within the superlattice, the SmN spin and orbital moments are not fully polarized.

A more quantitative analysis of the observed ED and EQ enhancement factors can also be made. The strong intra-ion $4 f-5 d$ exchange mechanism of the rare-earths means that inter-ion $4 f-4 f$ exchange proceeds via the $5 d$ states, resulting in an effective $4 f$ inter-ion exchange constant $(\mathcal{J})$ proportional to the $\mathrm{Sm} 5 d$ polarization given by the ED XMCD. From this identification it is clear that the average superlattice $\mathrm{SmN}$ exchange constant $\mathcal{J}_{S L}$ is enhanced by a factor of 3.1 (the ED enhancement factor) over the bulk $\mathrm{SmN}$ exchange $\mathcal{J}$. We can then calculate the expectation values for the superlattice $\mathrm{SmN}$ using $\mathcal{J}_{S L}=3.1 \times \mathcal{J}$ as the exchange constant. The enhancement of the orbital and spin moments in the superlattice compared to bulk $\mathrm{SmN}$ is then determined by the ratios $\mathcal{R}_{L}=\left\langle L_{z}\right\rangle_{\mathcal{J}_{S L}} /\left\langle L_{z}\right\rangle_{\mathcal{J}}$ and $\mathcal{R}_{S}=\left\langle S_{z}\right\rangle_{\mathcal{J}_{S L}} /\left\langle S_{z}\right\rangle_{\mathcal{J}}$. The ratios are plotted as a function of $\mathcal{J}$ in the third panel of Figure 2. The results show that for $\mathcal{J}=7.9 \mathrm{~K}$ and $\mathcal{J}_{S L}=24.5 \mathrm{~K}$ (see also Table II), values of $\mathcal{R}_{L}=1.56$ and $\mathcal{R}_{S}=1.52$ are returned, closely reproducing the factor of 1.6 enhancement in the EQ signal. As noted above, 


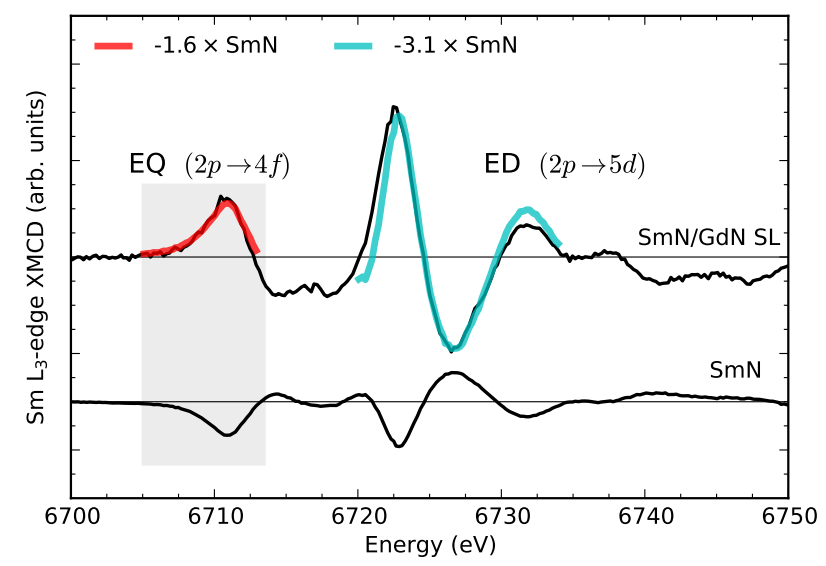

FIG. 3. $\mathrm{Sm} \mathrm{L}_{3}$ edge XMCD data from Ref. [15], showing spectra taken at $15 \mathrm{~K}$ and $6 \mathrm{~T}$ for $\mathrm{SmN}$ and a $\mathrm{SmN} / \mathrm{GdN}$ superlattice. The scaling of EQ and ED signals between samples is also displayed.

$\left\langle S_{z}\right\rangle$ and $\left\langle L_{z}\right\rangle$ scale nearly linearly with the EQ spectrum, demonstrating that the model describes the values if moments quite well.

\section{DISCUSSION}

The ability of the model to reproduce not only the net experimental moment in bulk SmN (sign and magnitude), but also the change in $\left\langle S_{z}\right\rangle$ and $\left\langle L_{z}\right\rangle$ due to interface exchange in the superlattice shows it captures the essential features of the $\mathrm{SmN}$ ground state. The results further show that contributions to the net moment from conduction electrons and other sources provide only small corrections to our results. The moderate quenching of the spin and orbital moments to magnitudes of $\sim 2 \mu_{B}$ in bulk SmN, enhanced to $\sim 3 \mu_{B}$ in SmN layers within the $\mathrm{SmN} / \mathrm{GdN}$ superlattice are significantly less than the values given by $\mathrm{LSDA}+U$ calculations. However, this quenching in SmN fits in with recent experiments 47. on HoN, ErN and DyN (all heavy rare-earth and spindominant systems) which also display significant reduction of the net moment compared to the Hund's rules values and the LSDA $+U$ calculations in Ref [25]. There the authors suggest the small ordered moments can be explained by full quenching of $\left\langle L_{z}\right\rangle$, but this seems unlikely due to the large spin-orbit coupling energy of the $4 f$ shell. It is more likely that in these RENs partial quenching of both $\left\langle S_{z}\right\rangle$ and $\left\langle L_{z}\right\rangle$ takes place, as we have shown for $\mathrm{SmN}$. The quenching of moments in rare-earth systems with small exchange energies due to crystal fields has been known for some time [48, suggesting that densityfunctional theory calculations in the RENs should be revisited to obtain more accurate descriptions of the magnetic structure.
The values of the spin and orbital moments of $\approx 2 \mu_{B}$ in bulk $\mathrm{SmN}$ and moments of $\approx 3 \mu_{B}$ in the superlattice $\mathrm{SmN}$ layers are also relevant to the the recent observation of superconductivity coinciding with ferromagnetic order in $\mathrm{SmN}$ 16. Superconductivity at $T_{c} \approx 3 \mathrm{~K}$ was observed in heavily doped SmN film, while in a $12 \times(10 \mathrm{~nm} \mathrm{GdN} / 5 \mathrm{~nm} \mathrm{SmN})$ superlattice a superconducting transition occurred at $T_{c} \approx 5 \mathrm{~K}$, with a much larger critical field. The heavy doping is expected to reduce the net moment (the conduction electron contribution $m_{\sigma}$ is parallel to $\left.m_{S}^{4 f}\right)$, and could even lead to a spin-dominant moment at high enough doping levels. The more robust superconductivity (which was shown to involve $4 f$ states) in the superlattice is likely due to enhancement of the $4 f$ spin and orbital moments due interface exchange with GdN. The enhanced $4 f$ moments may then be more robust against disorder, leading to a higher $T_{c}$ and a larger critical field. We note that the observed $4 f$ enhancement from XMCD data of Ref. [15] given in Fig. 3 is from $\mathrm{SmN} 1.5 \mathrm{~nm}$ thick, while the superconducting $\mathrm{SmN}$ layers in the $\mathrm{SmN} / \mathrm{GdN}$ superlattice of Ref. [16] were $5 \mathrm{~nm}$ thick, indicating that the effect of interface exchange is smaller (averaged over the $\mathrm{SmN}$ layers) in the superconducting samples, so that the average enhancement in $4 f$ polarization is probably less than $60 \%$ enhancement observed in the XMCD data.

Also closely related to the current calculations is the twisted magnetization phase observed in a SmN/GdN bilayer [15, in which the $\mathrm{SmN}$ is exchange coupled to GdN and one interface, and results in a rotating magnetization as the $\mathrm{SmN}$ moments experience exchangeZeeman competition. This twisted magnetization phase may be viewed as a type of unconventional exchangespring; it is the magnetically hard $\mathrm{SmN}$ layer which develops the spring (or twist), while the soft GdN layer is fixed. The $\mathrm{SmN} / \mathrm{GdN}$ system is also semiconducting, in contrast to conventional exchange spring-systems which are metallic. These features provide an opportunity for tunneling magnetoresistance experiments which may show unusual behavior. The current calculations revealing quenched moments will thus provide an important aide in the quantitative interpretation of tunneling magnetoresistance experiments involving $\mathrm{SmN} / \mathrm{GdN}$ heterostructures, and should allow for more accurate micromagnetic simulations of the twisted magnetization phase.

\section{CONCLUSION}

We have shown that the small orbital-dominant SmN moment can be explained by consideration of an effective $4 f$ Hamiltonian incorporating excited $J$-multiplets, exchange in a self-consistent mean-field approach, spinorbit coupling, and crystal field terms with parameters estimated from the point-charge model. The magnitudes of the spin and orbital moments were shown to be par- 
tially quenched to $\approx 2 \mu_{B}$ in bulk SmN. By linking the Sm $5 d$ XMCD polarization to the effective exchange constant, we showed that our model reproduces the enhancement in the Sm $4 f$ electric-quadrupole XMCD signal in the $\mathrm{SmN} / \mathrm{GdN}$ superlattice compared to bulk $\mathrm{SmN}$, due to the enhanced spin and orbital moments of magnitude $\approx 3 \mu_{B}$. The magnitudes of these moments are far less than values obtained from free-ion or LSDA $+U$ calculations, and suggests that theoretical band structure investigations of $\mathrm{SmN}$ and other rare-earth nitrides should be revisited. The partially quenched spin and orbital moments in $\mathrm{SmN}$ and their enhancement in the $\mathrm{SmN} / \mathrm{GdN}$ superlattice corroborate the previous report that the $4 f$ states are crucial for superconductivity in SmN. Finally, the values of the magnetic moments presented here will aide in quantitative interpretation of future tunelling magnetoresistance experiments involving $\mathrm{SmN}$ and $\mathrm{SmN} / \mathrm{GdN}$ heterostructures.

\section{APPENDIX}

The magnetic susceptibility $\chi$ may be written in terms of the susceptibility in the absence of exchange, $\chi_{0}$, and the molecular field constant $\lambda$ (in the mean-field approximation) as

$$
\frac{1}{\chi}=\frac{1}{\chi_{0}}-\lambda=\frac{1}{\chi_{C}+\chi_{V V}}-\lambda,
$$

where $\lambda=(V / N) 2 \mathcal{J}\left(g_{J}-1\right)^{2} /\left(g_{J} \mu_{B}\right)^{2}$. The Curie susceptibility is

$$
\chi_{C}=\frac{C}{T}=\frac{N}{V} \frac{g_{J}^{2} \mu_{B}^{2} J(J+1)}{3 k_{B} T}
$$

and the van Vleck susceptibility for $\mathrm{Sm}^{3+}$ is

$$
\chi_{V V}=\frac{N}{V} \frac{20 \mu_{B}^{2}}{7 \Delta E} .
$$

The presence of $\chi_{V V}$ modifies the usual Curie-Weiss temperature $\Theta=2 \mathcal{J}\left(g_{J}-1\right)^{2} J(J+1) / 3 k_{B}$, and thus our estimate of $\mathcal{J}$. To first order, the correction to the critical temperature $\Theta$ is given by the solution to $T=$ $\left(C+\chi_{V V} T\right) \lambda$. Solving for $\mathcal{J}$ yields

$$
\mathcal{J}=\frac{3}{2} \frac{k_{B} \Theta}{\left(g_{J}-1\right)^{2} J(J+1)}\left[\frac{1}{1+12 k_{B} \Theta / \Delta E}\right] .
$$

\section{ACKNOWLEDGEMENTS}

We acknowledge financial support from the New Zealand Foundation for Research, Science and Technology (Grant No. VICX0808) and the Marsden Fund (Grant No. 08-VUW- 030). The MacDiarmid Institute is supported by the New Zealand Centres of Research Excellence Fund.
[1] F. Natali, B. J. Ruck, N. O. V. Plank, H. J. Trodahl, S. Granville, C. Meyer, and W. R. L. Lambrecht, Prog. Mater Sci. 58, 1316 (2013)

[2] F. Leuenberger, A. Parge, W. Felsch, K. Fauth, and M. Hessler, Phys. Rev. B 72, 014427 (2005).

[3] S. Granville, B. J. Ruck, F. Budde, A. Koo, D. J. Pringle, F. Kuchler, A. R. H. Preston, D. H. Housden, N. Lund, A. Bittar, G. V. M. Williams, and H. J. Trodahl, Phys. Rev. B 73, 235335 (2006).

[4] A. R. H. Preston, S. Granville, D. H. Housden, B. Ludbrook, B. J. Ruck, H. J. Trodahl, A. Bittar, G. V. M. Williams, J. E. Downes, A. DeMasi, Y. Zhang, K. E. Smith, and W. R. L. Lambrecht, Phys. Rev. B 76, 245120 (2007)

[5] C. Meyer, B. J. Ruck, J. Zhong, S. Granville, A. R. H. Preston, G. V. M. Williams, and H. J. Trodahl, Phys. Rev. B 78, 174406 (2008).

[6] C. Meyer, B. J. Ruck, A. R. H. Preston, S. Granville, G. V. M. Williams, and H. J. Trodahl, J. Magn. Magn. Mater. 322, 1973 (2010)

[7] J. H. Richter, B. J. Ruck, M. Simpson, F. Natali, N. O. V. Plank, M. Azeem, H. J. Trodahl, A. R. H. Preston, B. Chen, J. McNulty, K. E. Smith, A. Tadich, B. Cowie, A. Svane, M. van Schilfgaarde, and W. R. L. Lambrecht, Phys. Rev. B 84, 235120 (2011).

[8] D. Le Binh, B. J. Ruck, F. Natali, H. Warring, H. J. Trodahl, E.-M. Anton, C. Meyer, L. Ranno, F. Wilhelm, and A. Rogalev, Phys. Rev. Lett. 111, 167206 (2013).

[9] M. Azeem, B. J. Ruck, D. Le Binh, H. Warring, H. J. Trodahl, N. M. Strickland, A. Koo, V. Goian, and S. Kamba, J. Appl. Phys. 113, 203509 (2013)

[10] H. Warring, B. J. Ruck, J. F. McNulty, E.-M. Anton, S. Granville, A. Koo, B. Cowie, and H. J. Trodahl, Phys. Rev. B 90, 245206 (2014).

[11] K. Senapati, M. G. Blamire, and Z. H. Barber, Nat. Mater. 10, 849 (2011)

[12] D. Massarotti, A. Pal, G. Rotoli, L. Longobardi, M. Blamire, and F. Tafuri, Nat. Commun. 6 (2015).

[13] H. Warring, B. Ruck, H. Trodahl, and F. Natali, Appl. Phys. Lett. 102, 132409 (2013).

[14] P. K. Muduli, A. Pal, and M. G. Blamire, Phys. Rev. B 89, 094414 (2014).

[15] J. F. McNulty, E.-M. Anton, B. J. Ruck, F. Natali, H. Warring, F. Wilhelm, A. Rogalev, M. M. Soares, N. B. Brookes, and H. J. Trodahl, Phys. Rev. B 91, 174426 (2015)

[16] E.-M. Anton, S. Granville, A. Engel, S. V. Chong, M. Governale, U. Zülicke, A. G. Moghaddam, H. J. Trodahl, F. Natali, S. Vézian, and B. J. Ruck, (2015), arXiv:1505.03621 [cond-mat]

[17] E.-M. Anton, B. J. Ruck, C. Meyer, F. Natali, H. Warring, F. Wilhelm, A. Rogalev, V. N. Antonov, and H. J. Trodahl, Phys. Rev. B 87, 134414 (2013)

[18] B. J. Ruck, H. J. Trodahl, J. H. Richter, J. C. Cezar, F. Wilhelm, A. Rogalev, V. N. Antonov, B. D. Le, and C. Meyer, Phys. Rev. B 83, 174404 (2011).

[19] H. De Wijn, A. Van Diepen, and K. Buschow, Phys. Status Solidi (B) 76, 11 (1976).

[20] H. Adachi, H. Ino, and H. Miwa, Phys. Rev. B 56, 349 (1997) 
[21] C. Mitra and W. R. L. Lambrecht, Phys. Rev. B 78, 134421 (2008)

[22] A. Sharma and W. Nolting, Phys. Rev. B 81, 125303 (2010)

[23] F. Natali, B. J. Ruck, H. J. Trodahl, D. L. Binh, S. Vezian, B. Damilano, Y. Cordier, F. Semond, and C. Meyer, Phys. Rev. B 87, 035202 (2013)

[24] C.-M. Lee, H. Warring, S. Vézian, B. Damilano, S. Granville, M. Al Khalfioui, Y. Cordier, H. J. Trodahl, B. J. Ruck, and F. Natali, Appl. Phys. Lett. 106, 022401 (2015).

[25] P. Larson, W. R. L. Lambrecht, A. Chantis, and M. van Schilfgaarde, Phys. Rev. B 75, 045114 (2007)

[26] C. Morari, F. Beiuşeanu, I. Di Marco, L. Peters, E. Burzo, S. Mican, and L. Chioncel, J. Phys.: Condens. Matter 27, 115503 (2015)

[27] L. Peters, I. Di Marco, P. Thunström, M. I. Katsnelson, A. Kirilyuk, and O. Eriksson, Phys. Rev. B 89, 205109 (2014)

[28] A. M. van Diepen, H. W. de Wijn, and K. H. J. Buschow, Phys. Rev. B 8, 1125 (1973)

[29] K. H. J. Buschow, A. M. van Diepen, and H. W. de Wijn, Phys. Rev. B 8, 5134 (1973)

[30] H. Adachi and H. Ino, Nature 401, 148 (1999)

[31] H. Adachi, H. Kawata, H. Hashimoto, Y. Sato, I. Matsumoto, and Y. Tanaka, Phys. Rev. Lett. 87, 127202 (2001)

[32] S. Qiao, A. Kimura, H. Adachi, K. Iori, K. Miyamoto, T. Xie, H. Namatame, M. Taniguchi, A. Tanaka, T. Muro, S. Imada, and S. Suga, Phys. Rev. B 70, 134418 (2004)

[33] S. S. Dhesi, G. van der Laan, P. Bencok, N. B. Brookes, R. M. Galéra, and P. Ohresser, Phys. Rev. B 82, 180402 (2010)
[34] H. Adachi, H. Ino, and H. Miwa, Phys. Rev. B 59, 11445 (1999).

[35] K. Stevens, Proc. Phys. Soc. London, Sect. A 65, 209 (1952).

[36] M. T. Hutchings, Solid State Physics 16, 227 (1964).

[37] We have found $z$ parallel to [111] to be the lowest energy orientation compared to [001] and [110] directions. This was also reported in 29] and 34. for $\mathrm{Sm}^{3+}$.

[38] R. J. Birgeneau, E. Bucher, J. P. Maita, L. Passell, and K. C. Turberfield, Phys. Rev. B 8, 5345 (1973).

[39] F. Hulliger, Handbook on the Physics and Chemistry of Rare Earths 4, 153 (1979).

[40] K. Lea, M. Leask, and W. Wolf, J. Phys. Chem. Solids 23, 1381 (1962).

[41] A. J. Freeman and R. E. Watson, Phys. Rev. 127, 2058 (1962)

[42] A. Freeman and J. Desclaux, J. Magn. Magn. Mater. 12, 11 (1979).

[43] To less than $1 \%$ error one may scale both $\left\langle r^{4}\right\rangle$ and $\left\langle r^{6}\right\rangle$ by 1.2 to go from Hartree-Fock to Dirac-Fock values, which is equivalent to rescaling $Z$. One must therefore scale $Z$ accordingly when comparing to values from the literature.

[44] B. T. Thole, P. Carra, F. Sette, and G. van der Laan, Phys. Rev. Lett. 68, 1943 (1992)

[45] P. Carra, B. T. Thole, M. Altarelli, and X. Wang, Phys. Rev. Lett. 70, 694 (1993).

[46] P. Carra, H. König, B. Thole, and M. Altarelli, Physica B: Condensed Matter 192, 182 (1993).

[47] D. L. Cortie, J. D. Brown, S. Brück, T. Saerbeck, J. P. Evans, H. Fritzsche, X. L. Wang, J. E. Downes, and F. Klose, Phys. Rev. B 89, 064424 (2014).

[48] G. T. Trammell, Phys. Rev. 131, 932 (1963). 